\title{
Fully Parametric Optimization Designs of Wing Components
}

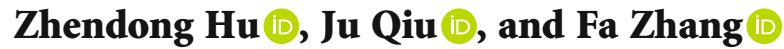 \\ Beijing Key Laboratory of Civil Aircraft Structures and Composite Materials, Beijing Aeronautical Science \& Technology Research \\ Institute of COMAC, Beijing 102211, China
}

Correspondence should be addressed to Fa Zhang; zhangfa@comac.cc

Received 16 April 2020; Revised 6 September 2020; Accepted 25 September 2020; Published 14 October 2020

Academic Editor: Seid H. Pourtakdoust

Copyright (C) 2020 Zhendong Hu et al. This is an open access article distributed under the Creative Commons Attribution License, which permits unrestricted use, distribution, and reproduction in any medium, provided the original work is properly cited.

\begin{abstract}
An optimization technique called shape-linked optimization, which is different from the traditional optimization method, is introduced in this paper. The research introduces an updated wing optimization design in an effort to adapt to continuous structure changes and shapes while optimizing for a lighter weight of the structure. The changing tendencies of the thickness of wing skins and the cross-section areas of the wing beams are fitted to continuous polynomial functions, whose coefficients are designed as variables, which is a different engineering approach from the size variants of the thickness and the area in the traditional optimization. The structural strength, stiffness, and stability are constraints. Firstly, this research unearths the significance of utilizing a modernized optimization process which alters the production of the traditional 12 or over 12 segment wing design and applies new approaches and methods with less variables that contribute to expedited design cycles, decreased engineering and manufacturing expenditures, and a lighter weight aircraft with lower operating costs than the traditional design for the operators. And then, this paper exemplifies and illustrates the validity of the above claims in a detailed and systematic approach by comparing traditional and modernized optimization applications with a two-beam wing. Finally, this paper also proves that the new optimized structure parameters are easier than the size optimization to process and manufacture.
\end{abstract}

\section{Introduction}

In the last century, the design of the wing was quite simple and inefficient, but now, the wing is a little light and generates significantly more lift with the less drag. More importantly, today's wings withstand a wider range of harsh flight conditions. How were these improvements developed? Wing design improvements, generally speaking, fell into three successive phases.

In the first phase, the flight loads of the wing were calculated by hand, and the structural strength or stiffness was checked manually. The previous engineers typically considered the spanwise and chordwise aerodynamic loads, according to Figure 1.

As can be seen, the wing lift distribution along the span was approximately elliptical, while chordwise was parabolic. The total forces of a single wing were defined by the following equation (1):

$$
L=\frac{3.5 \times g \times m_{\text {takeoff }}}{2} .
$$

Another limitation was that a wing structure was usually treated as a cantilever beam or an uncomplicated truss for more convenient stress analyses. In those years, the mechanics of material method employed by engineers was available for simple structural members subject to specific loadings such as axially loaded bars, prismatic beams in a state of pure bending, and circular shafts subject to torsion. The solutions could under certain conditions be superimposed using the superposition principle to analyze a member undergoing combined loading. Some experts started to use optimization method to do their design tasks.

Rao [1-3] introduced optimization of airplane wing structures under landing, gust, and taxiing loads. And a methodology had been presented for the automated optimum design of airplane wing structures under three types of loads. The procedure was demonstrated by considering the design of two example wings: one based on simple beam-type analysis and the other based on finite element analysis. The procedure was expected to be useful in the preliminary design stages. 


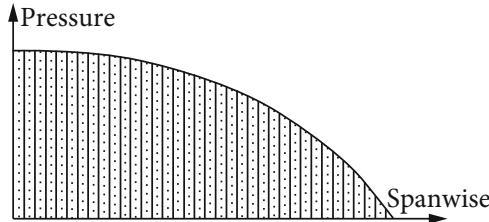

(a)

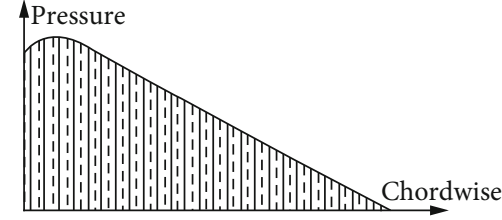

(b)

FIgURE 1: Spanwise load distribution (a) and chordwise load distribution (b). Unit: Pa.

In the second stage, with the early use of computers, engineers exploited programs to compute the aerodynamic load by $2 \mathrm{D}$ methods only, such as Double-lattice Method, due to restrictions in available computer memory, and access time to facilities. Rough experiments in wind tunnels allowed them to supplement the computational data. In structural analysis, elasticity methods were becoming available for simple structures using numerical approximation methods, while the Finite Element Method was commonly used to complicated structures. The pre- and postprocessing tools, such as aerodynamic computation or structural analysis, were almost nonexistent. During this period, engineers had known how to manually revise the contours or structures of the wings to better satisfy the design requirements, and many iterations helped them find the optimal design. At that time, lots of experts had many tries to optimize the structures.

Joo-Ho Choi (2002) addressed the shape design sensitivity analysis of a plane arch structure based on the variational formulation of a curved beam in linear elasticity. The sensitivity expression derived using the material derivative concept was very general and could thus be applied to complex arch shapes and their variation in a general direction. This method should be suitable for the aircraft beam's design.

Weigang and Weiji [4] thought if posterior preference optimization algorithm was used to solve this problem, the huge time consumption would be unacceptable in engineering practice due to the large amount of evaluation needed for the algorithm. So, a new interactive optimization algorithm, i.e., interactive multiobjective particle swarm optimization (IMOPSO), was presented in their work. It was worth extending in aviation.

James et al. [5] described that their work was unique in that the working domain of the design problem was given by the full three-dimensional region inside the wing skin, with no assumptions being made with regard to the number, location, or orientation of the structural members. It introduced the topology technology for an independent wing.

In the current phase, advanced computers are used throughout the world, so they are no longer a significant limiting factor. In preprocessing, engineers can quickly establish high fidelity models to simulate the wing's contour and structures directly using computers. The increased reliability of the data input naturally implies a more reliably computed solution. After calculations, postprocessing now offers detailed resultant images and vivid animations. In addition, both the top-ranking wind tunnel and CFD (Computational Fluid Dynamics) methods, which are widely applied to solve the aerodynamic loads by using Euler equations or Navier-Stokes equations, have the ability to provide better information for aerodynamic loads to guide design development. Finite element technology for structural analysis is also now sophisticated enough to handle just about any geometric shape wings made of advanced composite materials as long as sufficient computing power is available. Its applicability includes, but is not limited to, linear, nonlinear analysis, solid-fluid interactions, materials that are isotropic, orthotropic, or anisotropic, and external effects, consisting mainly of static, dynamic, thermodynamic, and environmental factors. As the optimization methods are proposed and the computing capability of the computers rapidly enhances, the optimization technology for wings develops by leaps and bounds. During this period, so many experts have done more optimization works by advanced optimization technologies.

Ronzheimer et al. [6] achieved a good goal to optimize the performance of a regional transport aircraft using high fidelity CFD- and CSM-methods. The geometrical inputs for the disciplines CFD and CSM were generated by CATIA V5 based on those design parameters which were prescribed by a SUBPLEX optimizer. CFD was at first used to calculate the drag in cruise flight with RANS and secondly to provide aerodynamic forces from Euler solutions from certain maneuver cases for a structural sizing of the wing to yield the wing weight. As with the structural calculations the wing deformations were available, these were used to deform the CFD mesh and to evaluate drag and forces on the corresponding flight shapes. This method may be used to the wing design.

Oktay, Akay, and Merttopcuoglu [7] used a structural topology optimization algorithm by using fluid-structure interaction method to account for flow-induced forces as in the case of air vehicles. The topology optimization tool used for design was the material distribution technique. Because reducing the weight requires numerous calculations, the CFD and structural optimization codes were parallelized and coupled via a code/mesh coupling scheme. In their study, the optimum rib topology had been determined for the concept phase.

Kenway and Martins [8] introduced multipoint highfidelity aerostructural optimization of a transport aircraft configuration.

Liu et al. [9] showed that the integrated global-local optimization approach had been applied to subsonic NASA common research model (CRM) wing, which proved the methodology's application scaling with medium fidelity FEM analysis. Both the global wing design variables and local panel design variables were optimized to minimize the wing weight at an acceptable computational cost. The structural weight of the wing had been, therefore, reduced by $40 \%$, and the parallel implementation allowed a reduction in the CPU time by $89 \%$. 
Boopathy, Rumpfkeil, and Kolonay [10] demonstrated structural sizing optimizations of a fighter wing configuration in the presence of uncertainties in structural parameters and material properties. And the design variables and input parameters were considered to have uncertainties and were treated as aleatory and epistemic random variables in the optimization process. They also indicated that a robust optimization framework under mixed epistemic and aleatory uncertainties using surrogate models for an application of interest to aircraft structural engineers.

Andrews and Perez [11] performed a multidisciplinary analysis which examined the aerodynamic performance of a box-wing regional jet aircraft throughout its mission and used a fully stressed beam analysis to examine the structure of the wing in detail.

Pahange and Abolbashari [12] investigated the numerical modeling of bird strike on an aircraft wing leading edge structure and tried to minimize simultaneously structural mass and wing skin deformation. They found that the influence of dimensions of wing internal structural components on the wing's damage after the collision with a bird was also studied. In this way, a low-weight leading edge structure to resist bird strike incidents was sought.

Dou and Jensen [13] extended the current structural optimization procedure to the more general case of modal analysis of nonlinear mechanical systems. The iterative optimization procedure consisted of calculation of nonlinear normal modes, solving an adjoint equation system for sensitivity analysis, and an update of design variables using a mathematical programming tool. Also, they demonstrated the method with examples involving plane frame structures where the hardening/softening behavior was qualitatively and quantitatively tuned by simple changes in the geometry of the structures.

Hernández et al. [14] showed that the use of the Phase Resonance Method or so-called Normal Mode Testing had been used for GVT of large aircrafts, which essentially consisted of applying single sine excitations at the structural natural frequencies.

Aage et al. [15] showed an amazing phenomenon, through the topological optimization of the wing structure. The optimized wing structure is similar to the biological skeleton that can bear loads in nature. This is the result of millions of years of evolution of nature, which conforms to the law of nature. The machine design, e.g., aircraft structures, also obeys it.

Winklberger et al. [16] introduced three configurations with different thread insert lengths and positions that were tested and compared. Their detailed numerical stress analysis showed that the hoop stress in the surrounding tube of the threaded connection was at the maximum, which might cause crack initiation and further lead to failure of the tie-rod. Finally, they made a conclusion that the stress concentration amplitude of the configuration with the highest fatigue life showed the lowest values at the open end of the tube.

Wang et al. [17] obtained results from the integrated optimization which provided designers with a wealth of information in the preliminary phase and important references for further design. A multidisciplinary optimization research of aerodynamics/structure/stability for a large airplane in a detailed design phase had already been performed in the study.
Zhao and Kapania [18] introduced bilevel programming weight minimization of composite flying-wing aircraft with curvilinear spars and ribs. Zhang and Xu [19] demonstrated the two-stage hybrid optimization, combined with the Taguchi-based grey relational optimization and NSGA-II based on surrogated model, was proposed to achieve design of honeycomb-type cellular structures under out-of-plane dynamic impact. Long et al. [20] proposed an efficient decomposition-based optimization framework using adaptive metamodelling for expensive aero-structure coupled wing optimization problems. The aero-structure coupled optimization problem was decomposed into $2 \mathrm{D}$ airfoil optimization and $3 \mathrm{D}$ wing optimization. Using the optimized airfoil, the wing optimization stage was further decomposed into system-level optimization and subsystem-level optimization. The proposed method was demonstrated on aerostructure coupled optimization of a high aspect ratio wing.

A mechanism/structure/aerodynamic multidisciplinary optimization platform based on the iSIGHT software was constructed for this smart high-lift system by Tian et al. [21]. Rajpal, Kassapoglou, and De Breuker [22] introduced aeroelastic optimization of composite wings including fatigue loading requirements. Zhao and Kapania [18] indicated bilevel programming weight minimization of composite flying-wing aircraft with curvilinear spars and ribs. Farsadi and Asadi [23] showed sequential quadratic optimization of aeroelastic energy of twin-engine wing system with curvilinear fiber path.

Recent years, for the complex optimization problem with different disciplines and a large number of design variables, a considerably high-dimensional design space is required, which creates an exponential challenge for the optimization. If we focus on building up a real model of the structure and other disciplines adopt high-fidelity surrogate models, it will be time-saving and dimension-reducing.

Unal, Lepsch, and McMillin [24] discussed response surface methods for approximation model building techniques which were central composite designs, minimum point designs, and overdetermined D-optimal designs for deterministic experiments. Ragon et al. [25] presented bilevel design of a wing structure using response surfaces. Kolonay and Kobayashi [26] introduced optimization of aircraft lifting surfaces using a cellular division method.

This paper uses a weighted least squares to fit continuous functions to express discrete structures, such as skin thickness using quadratic space function, beam's cross-section properties in local coordinates using one dimensional quadratic space function, and the coefficient of functions are defined as design variables, which can greatly reduce them, i.e., the dimension reduction (see Design Study).

Generally, the research of structural optimization is divided into four levels, which are sizing optimization, shape optimization, topology optimization, and topography optimization. Our current optimization is between size optimization and shape optimization, and it can get optimized structures similar to those after topology optimization.

The following of the paper is organized in the different parts. The first one introduces the wing design and the experts' work in this field. The second one is the introduction of the optimization technique, the optimization parameters 

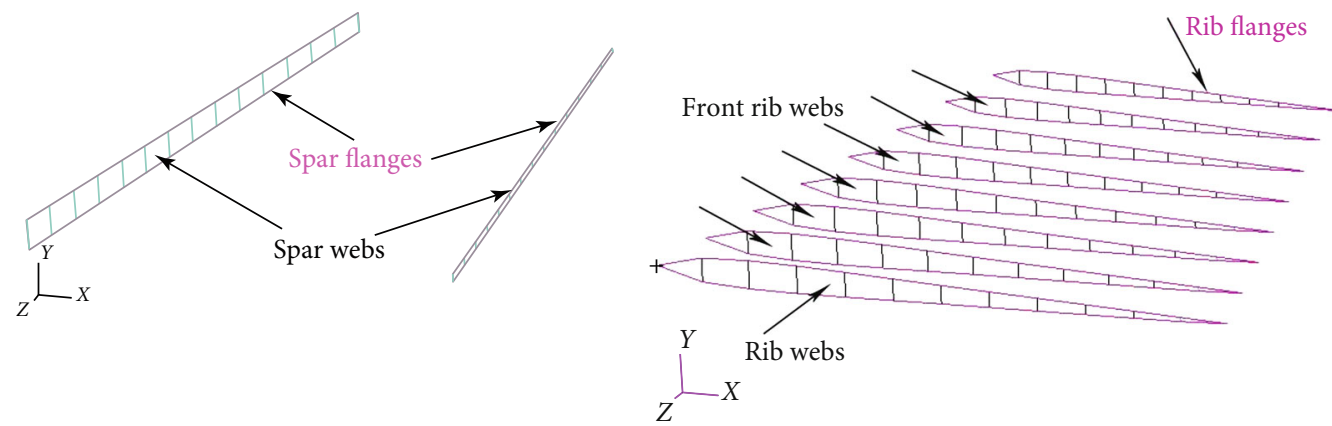

(a)

(b)

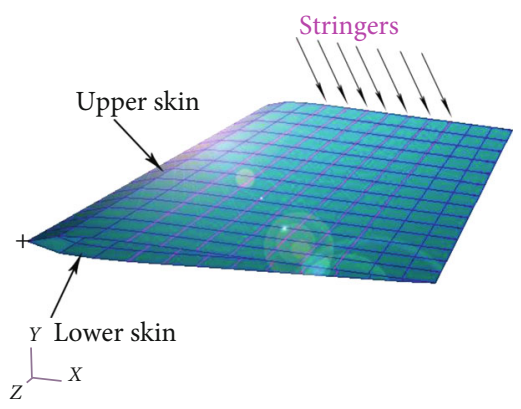

(c)

Figure 2: FEM of the wing (a-c).

of the wing, and discussion for optimization results, following this part, which is a summary section and future work.

\section{Main Section}

The main part introduces the whole optimization, including the selection of optimization methods, the definitions of the design variables, and constraints.

2.1. Optimization Algorithm and Response Surfaces. This section contains optimization principles, optimization methods, and the response surface.

2.1.1. Primary Optimization Equations. The typical optimization problem is given mathematically by equations (2) to (6):

$$
\text { Minimize : } F(x) \text { objective function. }
$$

Subject to : $p_{j}-1 \leq 0, j=1, m$ inequality constraints.

$$
\begin{aligned}
& h_{k}-1=0, k=1, l \quad \text { equality constraints. } \\
& X_{i}^{l} \leq X_{i} \leq X_{i}^{U}, i=1, n \quad \text { side constraints. }
\end{aligned}
$$

$$
X=\left\{\begin{array}{c}
X_{1} \\
X_{2} \\
\bullet \\
\bullet \\
\bullet \\
X_{n}
\end{array}\right\} \text { design variables. }
$$

This is the classic optimization problem statement. Stated in words, this says that it is desired to minimize an objective function subject to three types of constraints. According to the present study, the objective, design variables, and design constraints are listed in Design Study.

2.2. Pointer Algorithm. In iSIGHT platform (2008) [27], the Pointer is a global optimal tool. The Pointer technique consists of a complementary set of optimization algorithms: linear simplex, sequential quadratic programming (SQP), downhill simplex, and genetic algorithms (GA). In the process of optimization, linear simplex deals with the constant function; the best design is obtained quickly by use of SQP with good convergence and numerical stability near the peak of the problem; the downhill simplex method requires only function evaluations, not derivatives, but it may frequently be the best method to use if the figure of merit is get-something-working-quickly for a problem whose computational burden is small; GA is to extract optimization strategies nature uses successfully-known as Darwinian Evolutio$\mathrm{n}$-and transform them for application in mathematical optimization theory to find the global optimum in a defined phase space. The Pointer method organically combines four kinds of algorithms together and complements each other, which makes this analysis successful and efficient.

These optimization algorithms essentially can be classified into two groups: gradient-based methods and nongradient-based methods. The former ones determine the optimal design using the gradient information from a design sensitivity analysis. The recursive formulas of them are derived based on the Karush-Kuhn-Tucker (KKT) necessary conditions for an optimal design. SQP methods were developed for nonlinear gradient optimization in the last 
TABLE 1: Design variables of the wing.

\begin{tabular}{l} 
Design parts \\
\hline Thickness*1 \\
Upper and lower skin \\
Spar web \\
Rib web \\
Front rib web \\
A, $I_{1}, I_{2}, I_{12}, J^{* 2}$ \\
Spar flange \\
Rib flange (CROD) \\
Stringer \\
Ribs' spanwise percent \\
Spars' chordwise percent \\
${ }^{1}$ Thickness was from $0.005 \mathrm{~m}$ to $0.01 \mathrm{~m} .^{2} \mathrm{~A}$ was from $10^{-4} \mathrm{~m}^{2}$ to $10^{-6} \mathrm{~m}^{2} ; I_{1}$ \\
to $10^{-8} \mathrm{~m}^{4}$. \\
\end{tabular}

(a)

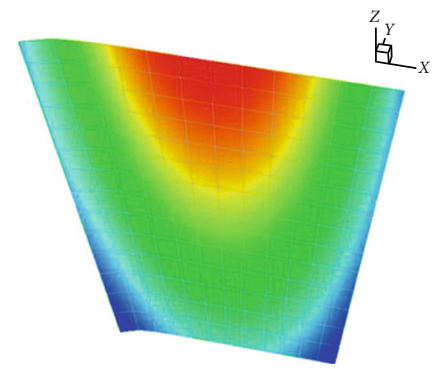

Thickness distribution of current design

(b)

FIGURE 3: Comparison of design variables of wings.

century. They are used for the problems where the objective function and the constraints are twice continuously differentiable. In order to reduce the computational cost, approximation concepts were constructed by experts. In combination with other techniques, such as constraint deletion, reciprocal approximation, and design variable linking, it has been successfully applied in structural optimization. It is, however, reported that the gradient-based methods normally find the optimal point close to the starting design point; in other words, it is possible to get a local optimum but not the global one. In terms of this weakness, the nongradient methods do not need gradient information at the design points. These methods contain the typical nature-inspired evolutionary methods, such as GA, which have recently demonstrated their success as well as popularity in engineering applications. Furthermore, the GA methods have been extensively applied in commercial aircraft wing optimization. Those methods are successful applications with the decentralized decision-making for exploiting the optimal design in the global design space.

Pointer can efficiently solve a wide range of problems in a fully automatic manner due to a special automatic control of both the gradient-based algorithm and nongradient algorithm. The goal of the Pointer technique is to make optimization more accessible to nonexpert users without sacrificing
Variables

Coefficients of 2 nd order polynomial in the variables $x, y$, and $z$

Coefficients of 2 nd order polynomial in the variables $x, y$, and $z$

Coefficients of piecewise linear polynomial in the variables $x, y$, and $z$ Lower and upper bound

Coefficients of 2nd order polynomial in the variables $x, y$, and $z$ Coefficients of piecewise linear polynomial in the variables $x, y$, and $z$ Coefficients of 2 nd order polynomial in the variables $x, y$, and $z$

Lower and upper bound

Lower and upper bound 


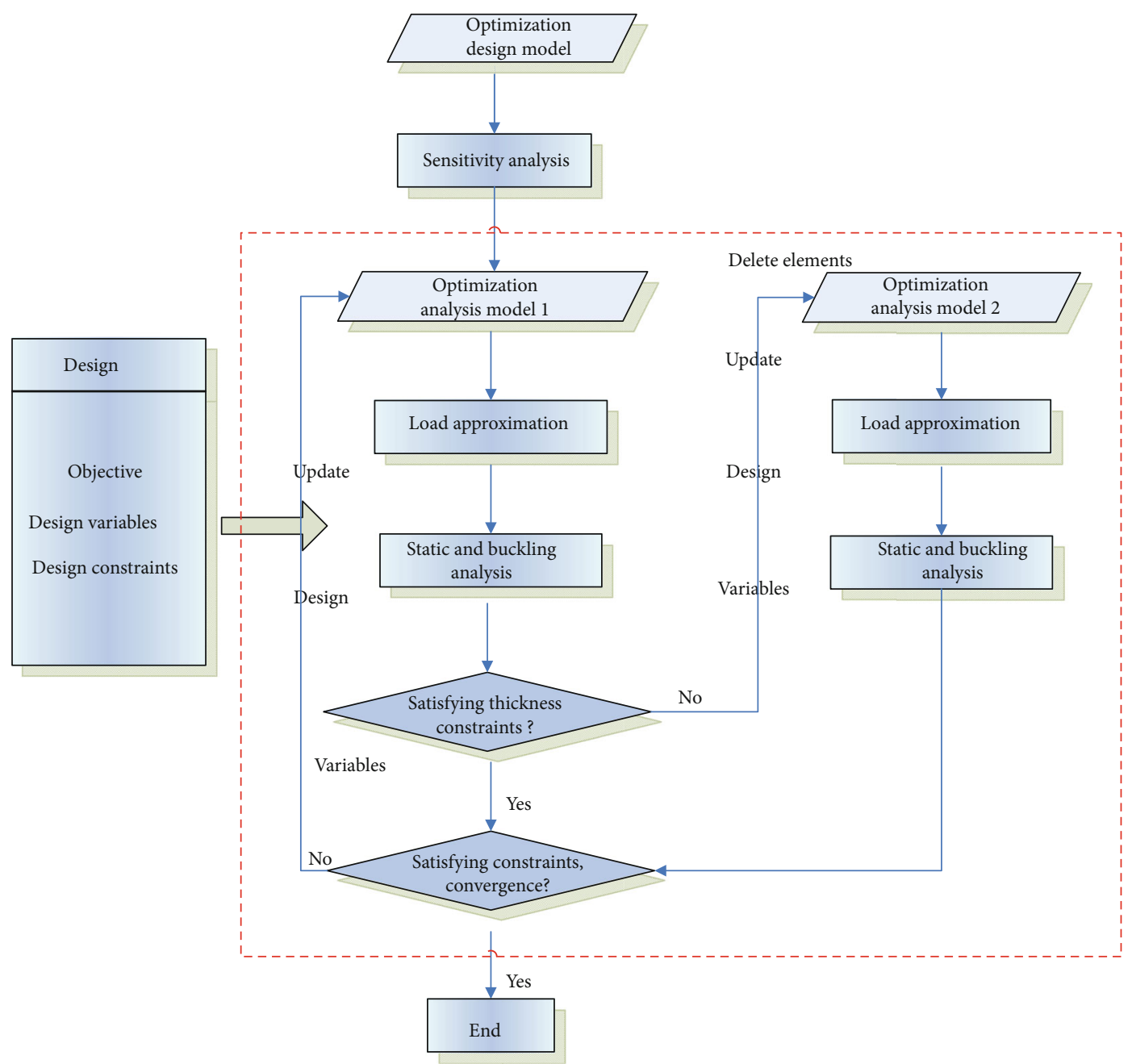

FIgURE 5: Flowchart of the wing optimization procedure.

optimization time. Surrogate models are worldwide used in the computational expensive optimizations, such as response surfaces optimization, Taylor series, neural networks method, and Kriging. In the present study, it uses computationally cheap hierarchical surrogate models to replace the exact and computationally expensive objective functions to reduce the computational cost.

Combining with central composite design (CCD) and curved surface fitting method, the response surface model of the flight load was constructed, and the mean square error (MSE) was used to judge the merits of the approximate model, modify the experimental design parameters, and adjust the scope of design variables, etc. The formulation (listed in equation (7)) of a response surface is defined using a second-order approximation function of the form.

$$
Y(X)=a_{0}+\sum_{i=1}^{n} b_{i} x_{i}+\sum_{i=1}^{n} c_{i i} x_{i}^{2}+\sum_{i j(i<j)}^{n} c_{i j} x_{i} x_{j}
$$

The polynomial coefficients of the skin and web's thickness and the beam properties were selected as inde- pendent variables. The coefficients of the aerodynamic loads were taken as dependent variables.

\subsection{Aircraft Wing}

2.4.1. Design Study. The aircraft wing can be decomposed into some panels that are bordered by the spars and ribs. The span of the wing is about $20 \mathrm{~m}$. The root and tip chords are $10 \mathrm{~m}$ and $6 \mathrm{~m}$, respectively. The airfoil thickness ratio is 0.0416 , and the sweep angle is $30^{\circ}$. As illustrated in Figure 2, the finite element model of the wing was established in the PCL functions of MSC [28]. This model consisted of 8 ribs, 2 spars, 7 stiffeners, and upper and lower surface skins. The front spar was positioned at $15 \%$ of chord and the rear spar at $85 \%$ of chord. 7 stringers were attached to the skin. Aluminum material was used for the entire model.

The wing design problem involves the wing's geometry dimension problems, for instance, spar and rib arrangements, etc. The plan view layout of an aircraft wing is to minimize its weight subject to constraints on stress, deflection, and buckling. It also is developed in conjunction with the thickness of the skin and web, the beam element with its 


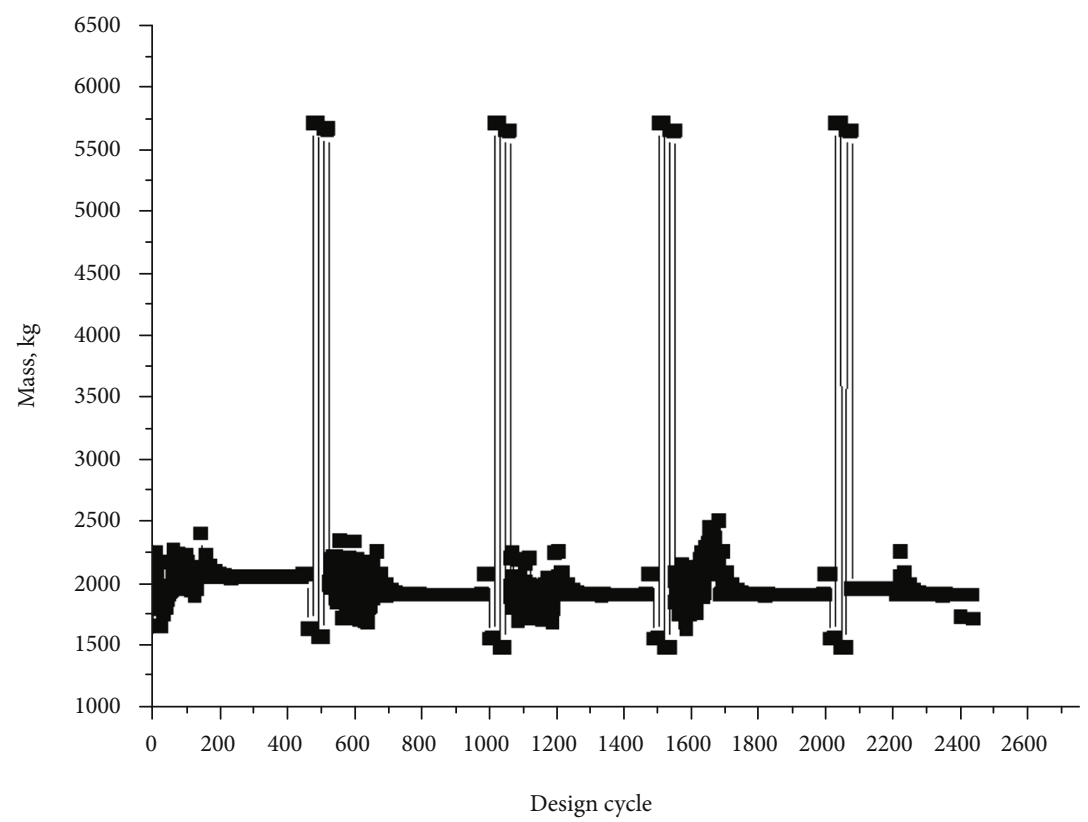

Figure 6: Optimization history.

cross-sectional area, moments of inertia, torsional constant, and the rod element for just cross-sectional area. Seven skin stringers are arranged between the front and rear spars.

The definition of each variable with respect to the wing's geometry is given in Table 1.

The locations of design variables are depicted in Figure 2.

According to the above definition, the number of design variables is diminished largely, for example, the plane and beam elements.

The traditional wing optimization design mainly includes $1 \mathrm{D}$ and 2D structure, which may have a partition thickness of more than 12 (see Figure 3), while the functional thickness expression proposed in this study has 10 coefficient design variables (see equation (8)). (8).

A complete space quadratic term is written in equation

$$
\begin{aligned}
f(X, Y, Z)= & A_{0}+A_{1}{ }^{*} X+A_{2}{ }^{*} Y+A_{3}{ }^{*} Z+A_{4}{ }^{*} X^{*} Y \\
& +A_{5}{ }^{*} X^{*} Z+A_{6}{ }^{*} Y^{*} Z+A_{7}{ }^{*} X^{*} X \\
& +A_{8}{ }^{*} Y^{*} Y+A_{9}{ }^{*} Z^{*} Z .
\end{aligned}
$$

As the design of the beam, there may be more than four sections in the span direction. See the following Figure 4 for the section size.

If we consider four segments, there are 6 design variables for each one. The total is 24 design variables. If taking crosssection area and moment of inertia, $A, I_{1}, I_{2}, I_{12}$, $J$, as design variables in the local coordinate system, which can be defined as one dimensional quadratic function listed in equation (9), we have a total of 15 variables, due to three coefficients in equation (9).

$$
f(X)=B_{0}+B_{1}{ }^{*} X+B_{2}{ }^{*} X^{*} X
$$

TABle 2: Comparisons of preoptimization and postoptimization location distribution and weight.

\begin{tabular}{lcccc}
\hline Conditions & Initial case & Case 1 & Case 2 \\
\hline Front spar & \multirow{2}{*}{ Chordwise percent (\%) } & 15.00 & 16.08 & 16.33 \\
Rear spar & 85.00 & 83.61 & 83.53 \\
\hline 1st rib & 0.00 & 0.00 & 0.00 \\
2nd rib & 14.29 & 14.76 & 14.70 \\
3rd rib & & 28.57 & 29.22 & 29.24 \\
4th rib & Spanwise percent (\%) & 42.86 & 43.39 & 43.34 \\
5th rib & & 57.14 & 57.32 & 57.47 \\
6th rib & 71.43 & 72.46 & 73.18 \\
7th rib & 85.71 & 86.95 & 87.94 \\
8th rib & 100.00 & 100.00 & 100.00 \\
\hline Mass (kg) & 1934.14 & 1711.47 & 1694.5 \\
\hline
\end{tabular}

It can be seen that there is a considerable reduction in design variables both in the 2-dimensional and 1dimensional elements.

The wing design problems to minimize its weight subject to constraints on stress, deflection, and buckling was summarized as follows:

$$
\begin{array}{cc}
\text { Minimize : } & \text { mass } \\
\text { Subject to : } & \sigma \leq 4.2 \mathrm{E} 8 \mathrm{~Pa} \\
& \gamma \leq 5^{0} \\
& f \geq 1.0 .
\end{array}
$$

Here, $\sigma$ is the yield stress for the material; $\gamma$ is the deflection angle of the tip wing; $f$ is the buckling factor.

The boundary condition for the wing was described as: 


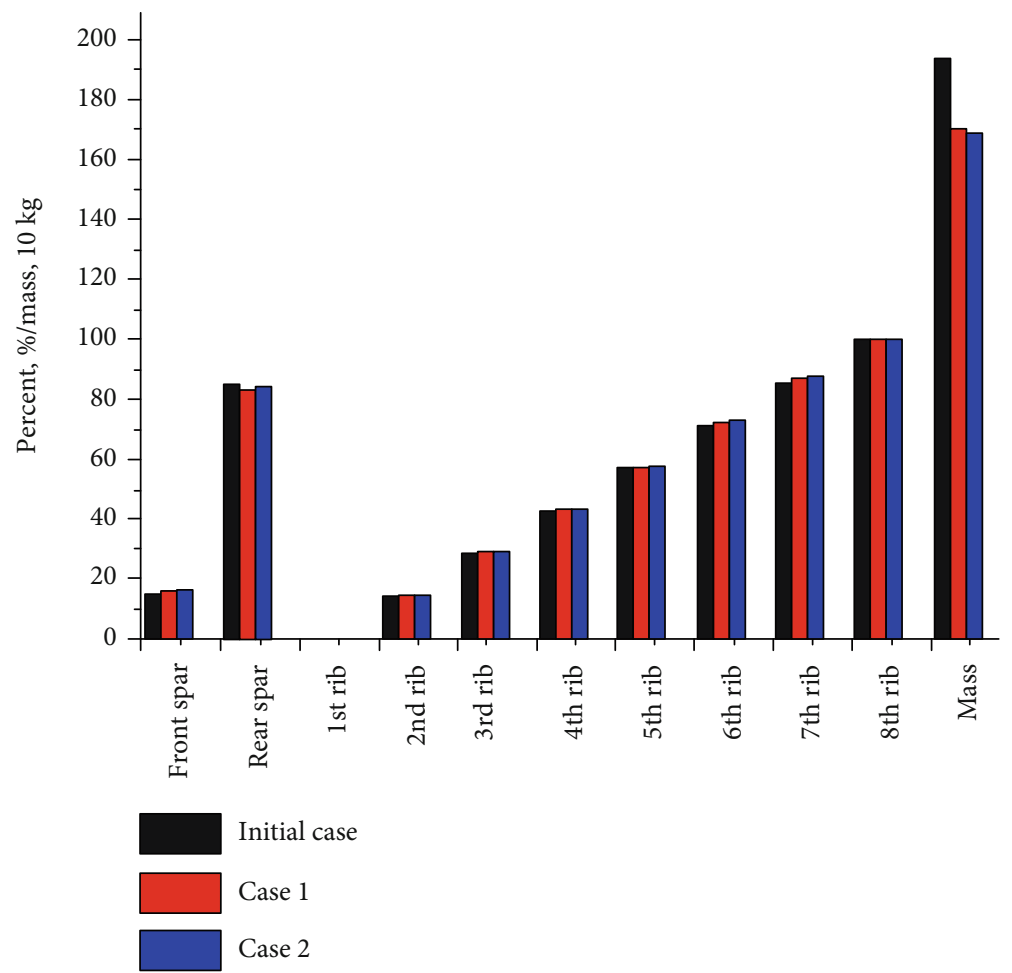

FIGURE 7: Comparisons of preoptimization and postoptimization conditions.
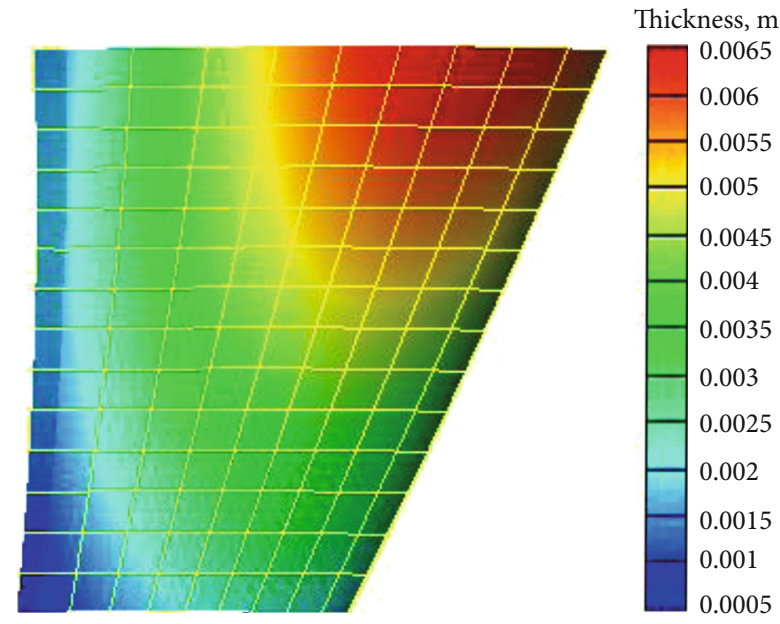

Figure 8: The thickness distribution of the upper skin (case 2).

The aerodynamic loads of surrogate model acted on the wing with all degrees of freedom fixed at the root.

Two cases of models were established. Note, case 1 referred to the wing with the front rib web; case 2 referred to the wing without the front rib web.

2.5. Design Procedure. In this paper, particularly, when the front rib web thickness was less than $0.0014 \mathrm{~m}$, the front rib web element would be temporarily deleted. The development of the optimization procedure is listed in Figure 5.

2.6. Numerical Results. The optimization history of the structural mass is shown in Figure 6. The number of the iteration

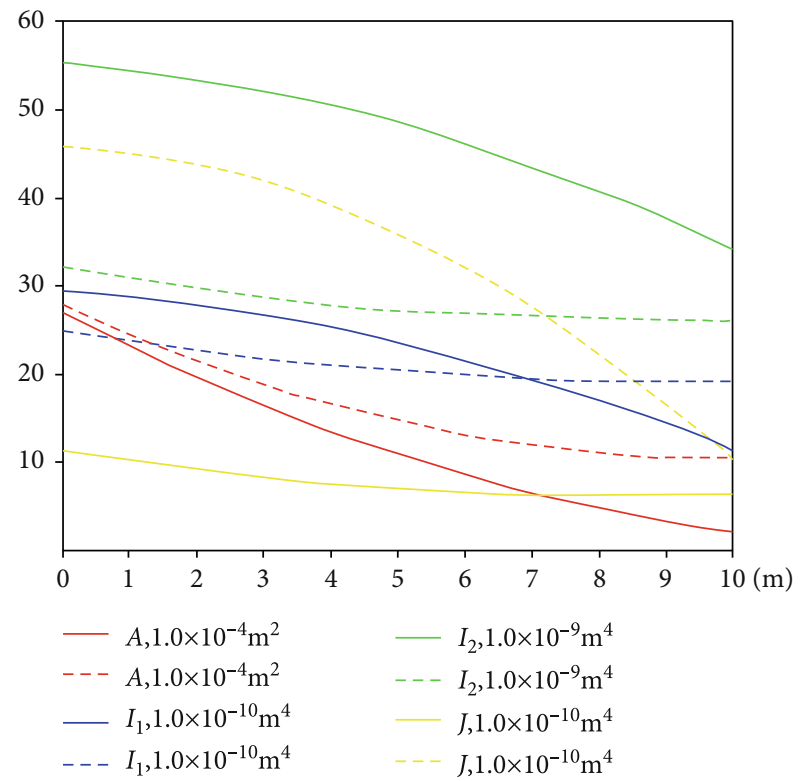

Figure 9: The section properties of the spar flange along the length of the spar (case 2).

is 2437. From the following historical curve, it appears to have repeated iterations due to the genetic algorithm's multidirectional search technique.

Table 2 presents preoptimization and postoptimization distribution of spars and ribs. The changing location of spars and ribs is depicted in Figure 7.

Table 2 and Figure 7 show that the differences between the spars and rib locations are small between the preoptimization and the postoptimization model. It indicates the initial 
arrangement of the wing is basically reasonable. As can be seen in Table 2, the mass of the wing in case 1 is $11.51 \%$ lower than that of the initial wing, while that in case 2 is a $12.39 \%$ weight reduction. This indicates that the deletion of the front ribs is of great benefit to weight loss.

The thickness distribution of the upper skin in postoptimization is shown in Figure 8. The trend is to become thinner as we move from the root to the tip.

Figure 9 depicts the section properties of the spar flange. The solid line refers to initial design; the dashed line refers to final design. Both the area and the moments of inertia decrease continuously from the root to tip.

\section{Conclusion}

The approach described in this paper has demonstrated that it could be capable reducing weight with continuous thickness and section property changes despite being a discretized model. In addition, the element deletion was also shown to benefits of the weight reduction. Unlike previous design efforts where the structures are traditionally partitioned to a lot of zones with numerous design variants, this work represents a very simple and high efficient approach with parametric expressions, coming of fewer variables. The proposed approach performs fully automatic modelling and loading. The parametric wing model as a primary design offers a good starting point for more detailed structural analysis. In a word, this approach is expected to widely be applied to most of the aircraft parts, such as horizontal tails, a vertical tail, and a fuselage.

Additionally, for the current design, almost all of the parts of the wing are defined as functions, which expresses continuous changes of thickness of the skin and properties of spars and stiffeners, and optimal results tend to do integrative processing. For another thing, another advantage of the successive structure distribution is that it has the lighter weight subject to the equal load. Commonly, this parametric structure design can be a further $1 \%$ through $3 \%$ wing weight decrease, compared with the traditional design (see Figure 3(a)).

Besides, a global optimal tool, including a combination group of optimization algorithms: linear simplex, sequential quadratic programming, downhill simplex, and genetic algorithms, which integrates the merits of global and local optimization, performs the optimization of the wing components. This avoids to miss the best point. And also, the approximate model is used to fit the aerodynamic loads for further time savings. All of the techniques to provide the primary commercial aircraft design are referenced.

All in all, the great thing of this proposal is that it can save a considerable amount of design time and costs.

\section{Nomenclature}

\section{Symbols}

A:

$a_{0}, b_{i}, c_{i i}, c_{i j}$ :

$F(x)$ :

$f:$

$\begin{array}{ll}g: & \text { Acceleration of gravity } \\ p: & \text { Inequality constraint function } \\ h: & \text { Equality constraint function } \\ I_{1}, I_{2}, I_{12}: & \text { Moments of inertia } \\ J: & \text { Torsional constant } \\ L: & \text { Total lift force } \\ l: & \text { Number of equality constraint function } \\ m: & \text { Number of inequality constraint } \\ n: & \text { function } \\ m_{\text {takeoff }}: & \text { Number of design variables } \\ X\left(x_{i}, x_{j}, i, j=1, n\right): & \text { Takeoff mass } \\ X^{l}: & \text { Design variables } \\ X^{u}: & \text { Lower bound side constraints } \\ Y(X): & \text { Apper bound side constraints } \\ \gamma: & \text { Wingtip deflection angle } \\ \sigma: & \text { von Mises stress } \\ A_{0}, A_{1}, \cdots, A_{9}: & \text { Functional coefficients } \\ B_{0}, B_{1}, B_{2}: & \text { Functional coefficients. }\end{array}$

\section{Definitions, Acronyms, and Abbreviations}

CFD: Computational Fluid Dynamics

CSM: Computational Structural Mechanics

RANS: Reynolds equations

SQP: Sequential quadratic programming

GA: Genetic algorithms.

\section{Data Availability}

The data used to support the findings of this study are included within the supplementary information file (s). (1) The node2.bdf data used to support the findings of this study have been used in building the wing model in MSC.Patran. This is a group of airfoil data, which was copy to MSC.Patran GUI for forming Patran's PCL (Patran3.ses). (2) The patran3.ses data used to support the findings of this study in Figure 5 are the building-mode file included within the article. This file described the process, that is, building-up wing model by MSC.Patran. (3) The ee5_right.bdf data used to support the findings of this study in Figure 5 are the solution file of MSC. Nastran included within the article. This file was obtained, after running the patran3.ses. (4) The gonastran. bat and gopatran. bat data used to support the findings of this study in Figure 5 are the executable files of Isight included within the article. gopatran. bat was used to call patran. exe to run patran3.ses in Isight, gonastran. bat was used to call nastran. exe to run ee5_right.bdf in Isight. (5) The test1.zmf data used to support the findings of this study in Figure 5 are the running file in Isight included within the article. This file was an executable file of Isight. (6) The Functional_equation data used to support the findings of this study in Figure 5 are the load function included within the article. This file was a fitted spatial function.

\section{Additional Points}

Further Work. These days, the traditional structural optimization is still size or topology optimization. This proposal 
method to optimize the structure in line with modern overall manufacturing needs to get the project to complete and verify the structural design as soon as possible, and we may further extend it to composite parts of aircraft.

However, for an all-composite wing, the stiffness of the metal beam is difficult to be equivalent to the stiffness of the composite one.

\section{Conflicts of Interest}

The authors declare that there is no conflict of interest regarding the publication of this paper.

\section{Acknowledgments}

The authors acknowledge the financial supports from the Special Project of Civil Aircraft of Ministry of Industry and Information Technology of China (Grant Number MJ2017-F-20). This method is patented by BASTRI. Authorized patent number is CN105528481 B. Authorized announce date is June 29th, 2018.

\section{References}

[1] S. S. Rao, "optimization of airplane wing structures under landing loads," Computers \& structures, vol. 19, no. 5-6, pp. 849-863, 1984.

[2] S. S. Rao, "Optimization of airplane wing structures under gust loads," Computers \& Structures, vol. 21, no. 4, pp. 741-749, 1986.

[3] S. S. Rao, "Optimization of airplane wing structures under taxiing loads," Computers \& Structures, vol. 26, no. 3, pp. 469-479, 1987.

[4] A. Weigang and L. Weiji, "Interactive multi-objective optimization design for the pylon structure of an airplane," Chinese Journal of Aeronautics, vol. 20, no. 6, pp. 524-528, 2007.

[5] K. A. James, J. R. R. A. Martins, and J. S. Hansen, "Threedimensional structural topology optimization of an aircraft wing using level set methods," in 12th AIAA/ISSMO Multidisciplinary Analysis and Optimization Conference, Victoria, British Columbia, Canada, September 2008.

[6] A. Ronzheimer, F. J. Natterer, and J. Brezillon, "Aircraft wing optimization using high fidelity closely coupled CFD and CSM methods," in 13th AIAA/ISSMO Multidisciplinary Analysis Optimization Conference, Fort Worth, TX, USA, September 2010.

[7] E. Oktay, H. U. Akay, and O. Merttopcuoglu, "Parallelized structural topology optimization and CFD coupling for design of aircraft wing structures," Computers \& Fluids, vol. 49, no. 1, pp. 141-145, 2011.

[8] G. K. W. Kenway and J. R. R. A. Martins, "Multipoint highfidelity aerostructural optimization of a transport aircraft configuration," Journal of Aircraft, vol. 51, no. 1, pp. 144-160, 2014.

[9] Q. Liu, M. Jrad, S. B. Mulani, and R. K. Kapania, "Integrated global wing and local panel optimization of aircraft wing," in 56th AIAA/ASCE/AHS/ASC Structures, Structural Dynamics, and Materials Conference, pp. 1-19, Kissimmee, FL, USA, January 2015 .
[10] K. Boopathy, M. P. Rumpfkeil, and R. M. Kolonay, "Robust optimization of a wing under structural and material uncertainties," in 17th AIAA Non-Deterministic Approaches Conference, pp. 1-20, Kissimmee, FL, USA, January 2015.

[11] S. A. Andrews and R. E. Perez, "Multidisciplinary analysis of a box-wing aircraft designed for a regional-jet mission," in 16th AIAA/ISSMO Multidisciplinary Analysis and Optimization Conference, pp. 1-14, Dallas, TX, USA, June 2015.

[12] H. Pahange and M. H. Abolbashari, "Mass and performance optimization of an airplane wing leading edge structure against bird strike using Taguchi-based grey relational analysis," Chinese Journal of Aeronautics, vol. 29, no. 4, pp. 934-944, 2016.

[13] S. Dou and J. S. Jensen, "Optimization of hardening/softening behavior of plane frame structures using nonlinear normal modes," Computers and Structures, vol. 164, no. 2016, pp. 63-74, 2016.

[14] S. Hernández, E. Menga, S. Moledo et al., "Optimization approach for identification of dynamic parameters of localized joints of aircraft assembled structures," Aerospace Science and Technology, vol. 69, no. 2017, pp. 538-549, 2017.

[15] N. Aage, E. Andreassen, B. S. Lazarov, and O. Sigmund, "Gigavoxel computational morphogenesis for structural design," Nature, vol. 550, no. 7674, pp. 84-86, 2017.

[16] M. Winklberger, P. Heftberger, M. Sattlecker, and M. Schagerl, "Fatigue strength and weight optimization of threaded connections in tie-rods for aircraft structures," Procedia engineering, vol. 213, no. 2018, pp. 374-382, 2018.

[17] X. Wang, Z. Wan, Z. Liu, and C. Yang, "Integrated optimization on aerodynamics-structure coupling and flight stability of a large airplane in preliminary design," Chinese Journal of Aeronautics, vol. 31, no. 6, pp. 1258-1272, 2018.

[18] W. Zhao and R. K. Kapania, "Bilevel programming weight minimization of composite flying-wing aircraft with curvilinear spars and ribs," AIAA Journal, vol. 57, no. 6, pp. 2594$2608,2019$.

[19] S. Zhang and F. Xu, "A two-stage hybrid optimization for honeycomb-type cellular structures under out-of-plane dynamic impact," Applied Mathematical Modelling, vol. 80, pp. 755-770, 2020.

[20] T. Long, Y. Wu, Z. Wang, Y. Tang, D. Wu, and Y. Yu, "Efficient aero-structure coupled wing optimization using decomposition and adaptive metamodeling techniques," Aerospace Science and Technology, vol. 95, article 105496, pp. 1-13, 2019.

[21] Y. Tian, J. Quan, P. Liu, D. Li, and C. Kong, "Mechanism/structure/aerodynamic multidisciplinary optimization of flexible high-lift devices for transport aircraft," Aerospace Science and Technology, vol. 93, article 104813, no. 93, pp. 1-22, 2019.

[22] D. Rajpal, C. Kassapoglou, and R. De Breuker, "Aeroelastic optimization of composite wings including fatigue loading requirements," Composite structures, vol. 227, no. 1, article 111248, 2019.

[23] T. Farsadi and D. Asadi, "Eğrisel Fiber Yollu İkili MotorluKanat Sisteminin Aeroelastik Enerji Optimizasyonu," Journal of Aviation, vol. 4, no. 1, pp. 1-14, 2020.

[24] R. Unal, R. A. Lepsch, and M. L. McMillin, "Response surface model building and multidisciplinary optimization using Doptimal designs," in 7th AIAA/USAF/NASA/ISSMO 
Symposium on Multidisciplinary Analysis and Optimization, pp. 1-7, St. Louis, MO, USA, September 1998.

[25] S. A. Ragon, Z. Gürdal, R. T. Haftka, and T. J. Tzong, "Bilevel design of a wing structure using response surfaces," Journal of Aircraft, vol. 40, no. 5, pp. 985-992, 2003.

[26] R. M. Kolonay and M. H. Kobayashi, "Optimization of aircraft lifting surfaces using a cellular division method," Journal of Aircraft, vol. 52, no. 6, pp. 2051-2063, 2015.

[27] Pointer Algorithm, "Isight user's guide," in 3DS Simulia, 2008.

[28] P. C. L. Patran, Patran Documentation, Macheal-Schwendler Corporation, Los Angeles, 2012. 